\title{
Interpolations and Fractional Sobolev Spaces in Carnot Groups
}

\author{
Ali Maalaoui ${ }^{(1)}$, Andrea Pinamonti ${ }^{(2)}$
}

\begin{abstract}
In this paper we present an interpolation approach to the fractional Sobolev spaces in Carnot groups using the K-method. This approach provides us with a different characterization of these Sobolev spaces, moreover, it provides us with the limiting behavior of the fractional Sobolev norms at the end-points. This allows us to deduce results similar to the Bourgain-Brezis-Mironescu and Maz'ya-Shaposhnikova in the case $p>1$ and Dávila's result in the case $p=1$. Also, this allows us to deduce the limiting behavior of the fractional perimeter in Carnot groups.
\end{abstract}

\section{Introduction}

Carnot groups appear as the first level extension of the classical Euclidean spaces, in the sense that they are modeled over $\mathbb{R}^{n}$ but with a different group structure. Nevertheless, they share many analytical properties with the Euclidean case. The typical example of Carnot group is the classical Heisenberg group. Lately, there have been a lot of interest in PDEs and fractional PDEs in this group coming from a geometric background since it is the flat context of CRgeometry, see for instance $[22,23,34,27]$ and the references therein. Moreover, Carnot groups have also been largely studied in several respects, such as differential geometry [13], subelliptic differential equations $[11,18,17,36]$ and complex variables [39]. For a general introduction to Carnot groups from the point of view of the present paper and for further examples, we refer, e.g., to $[11,18,39]$.

It is natural then to investigate to which extent one can generalize to Carnot groups the analytical tools that are well understood in the Euclidean case, see for instance [16, 28].

In this setting, we propose to study fractional Sobolev spaces from an interpolation point of view. Fractional Sobolev spaces in the literature, are also called Aronszajn, Gagliardo or Slobodeckij spaces, by the name of the ones who introduced them, almost simultaneously $[2,25,37]$. In Carnot groups fractional Sobolev spaces have been introduced and studied in $[18,17]$ and many different characterizations are now present, such as the ones in [35]. In the present paper we use the $K$-method for real interpolation, see for instance [4], to give an alternative characterization of fractional Sobolev spaces in Carnot groups. As a consequence, we derive a Bourgain-Brezis-Mironescu [5, 6, 7] (Theorem 5.1) and Ma'zya-Shapashnikova type limiting behavior (Theorem 5.2) of the Sobolev norms similarly to the approach developed in [29]. We point out that the exact limit of the fractional Sobolev norm (as the fractional parameter goes to

\footnotetext{
${ }^{1}$ Department of mathematics and natural sciences, American University of Ras Al Khaimah, PO Box 10021, Ras Al Khaimah, UAE. E-mail address: ali.maalaoui@aurak.ac.ae

${ }^{2}$ Dipartimento di Matematica, Universita di Trento, Via Sommarive 14, 38123 Povo, Trento, Italy. E-mail address: andrea.pinamonti@gmail.com
} 
1) was investigated in [3] using exact and technical computations. We also bring to the reader's attention the extensions of these type of results to other settings and to different functionals as in $[1,7,8,9,10,30,31,32]$. For $p=1$ we provide a limiting behavior leading to the space of $B V$-functions that are of great interest in geometric measure theory in the setting of Carnot groups, see $[19,20,21]$. This will allow us to characterize the fractional perimeter in Carnot groups and understand its limiting behavior when the fractional parameter goes to 1 , as it was done in the Euclidean setting in $[15,33]$.

This manuscript is structured as follows: First, in Section 2, we present the structure of Carnot groups and define Sobolev Spaces and $B V$-Spaces in this setting. In Section 3, we provide the necessary notations, definitions and properties of the K-interpolation, which will be the main tool in our investigation. In Section 4, we provide another characterization of the $K$ function in Carnot groups. This allows us to deduce an alternative characterization of the fractional Sobolev spaces. Finally, in Section 5, we provide applications of the characterizations given in Section 3. Namely, we present the limiting behavior of the Fractional Sobolev norms in the two end points, allowing us to obtain results similar to the ones already proved by BourgainBrezis-Mironescu and by Ma'zya-Shaposhnikova in $[5,6,7]$ for the case $p>1$ and by Davila in [15] for the case $p=1$. Also, we provide an alternative definition and characterization to the fractional perimeter and its limiting behavior at the end-points as in [15, 33].

\section{Carnot groups}

A connected and simply connected stratified nilpotent Lie group $(\mathbb{G}, \cdot)$ is said to be a Carnot group of step $k$ if its Lie algebra $\mathfrak{g}$ admits a step $k$ stratification, i.e., there exist linear subspaces $V_{1}, \ldots, V_{k}$ such that

$$
\mathfrak{g}=V_{1} \oplus \ldots \oplus V_{k}, \quad\left[V_{1}, V_{i}\right]=V_{i+1}, \quad V_{k} \neq\{0\}, \quad V_{i}=\{0\} \text { if } i>k,
$$

where $\left[V_{1}, V_{i}\right]$ is the subspace of $\mathfrak{g}$ generated by the commutators $[X, Y]$ with $X \in V_{1}$ and $Y \in V_{i}$.

Set $m_{i}=\operatorname{dim}\left(V_{i}\right)$, for $i=1, \ldots, k$ and $h_{i}=m_{1}+\cdots+m_{i}$, so that $h_{k}=n$. For sake of simplicity, we write also $h_{0}=0, m:=m_{1}$. We denote by $Q$ the homogeneous dimension of $\mathbb{G}$, i.e., we set

$$
Q:=\sum_{i=1}^{k} i \operatorname{dim}\left(V_{i}\right) .
$$

We choose now a basis $e_{1}, \ldots, e_{n}$ of $\mathbb{R}^{n}$ adapted to the stratification of $\mathfrak{g}$, i.e., such that $e_{h_{j-1}+1}, \ldots, e_{h_{j}}$ is a basis of $V_{j}$ for each $j=1, \ldots, k$. Moreover, let $X=\left\{X_{1}, \ldots, X_{n}\right\}$ be the family of left invariant vector fields such that $X_{i}(0)=e_{i}, i=1, \ldots, n$. The exponential mapping $\exp : \mathfrak{g} \rightarrow \mathbb{G}$ is a diffeomorphism. Given a basis $X_{1}, \ldots, X_{n}$ of $\mathfrak{g}$ adapted to the stratification, any $x \in \mathbb{G}$ can be written in a unique way as

$$
x=\exp \left(x_{1} X_{1}+\ldots+x_{n} X_{n}\right)=e^{x_{1} X_{1}+\ldots+x_{n} X_{n}} .
$$

We identify $x$ with $\left(x_{1}, \ldots, x_{n}\right) \in \mathbb{R}^{n}$ and hence $\mathbb{G}$ with $\mathbb{R}^{n}$. This is known as exponential coordinates of the first kind

The sub-bundle of the tangent bundle $T \mathbb{G}$ that is spanned by the vector fields $X_{1}, \ldots, X_{m}$ is called the horizontal bundle $H \mathbb{G}$; the fibers of $H \mathbb{G}$ are

$$
H_{x} \mathbb{G}=\operatorname{span}\left\{X_{1}(x), \ldots, X_{m}(x)\right\}, \quad x \in \mathbb{G} .
$$


We can endow each fiber of $H \mathbb{G}$ with an inner product $\langle\cdot, \cdot\rangle$ and with a norm $|\cdot|$ that make the basis $X_{1}(x), \ldots, X_{m}(x)$ an orthonormal basis. For any $\lambda>0$, the dilation $\delta_{\lambda}: \mathbb{G} \rightarrow \mathbb{G}$, is defined as

$$
\delta_{\lambda}\left(x_{1}, \ldots, x_{n}\right)=\left(\lambda \xi_{1}, \ldots, \lambda^{k} \xi_{k}\right),
$$

where $x=\left(\xi_{1}, \ldots, \xi_{k}\right) \in \mathbb{R}^{m_{1}} \times \ldots \times \mathbb{R}^{m_{k}} \equiv \mathbb{G}$.

The Haar measure of $\mathbb{G}=\left(\mathbb{R}^{n}, \cdot\right)$ is the Lebesgue measure in $\mathbb{R}^{n}$. If $A \subset \mathbb{G}$ is Lebesgue measurable, we write $|A|$ to denote its Lebesgue measure.

Once an orthonormal basis $X_{1}, \ldots, X_{m}$ of the horizontal layer is fixed, we define, for any function $f: \mathbb{G} \rightarrow \mathbb{R}$ for which the partial derivatives $X_{j} f$ exist, the horizontal gradient of $f$, denoted by $\nabla_{H} f$, as the horizontal section

$$
\nabla_{H} f:=\sum_{i=1}^{m}\left(X_{i} f\right) X_{i},
$$

whose coordinates are $\left(X_{1} f, \ldots, X_{m} f\right)$. If $\varphi=\left(\varphi_{1}, \ldots, \varphi_{m}\right) \in C_{c}^{1}\left(\mathbb{G}, \mathbb{R}^{m}\right)$ we put

$$
\operatorname{div}_{\mathbb{G}} \varphi=\sum_{i=1}^{m} X_{i} \varphi_{i} .
$$

Let $|\cdot|: \mathbb{G} \rightarrow[0, \infty)$ denote a symmetric homogeneous norm on $\mathbb{G}[11]$. Since any two continuous homogeneous norm are equivalent [11], from now on we denote by $|\cdot|$ any one of them. We denote by

$$
B_{r}(x)=\left\{y \in \mathbb{G}:\left|y^{-1} \cdot x\right|<r\right\}
$$

the ball centered at $x \in \mathbb{G}$ with radius $r>0$ and by $B_{r}=B(0, r)$.

We are now in position to introduce Sobolev and BV functions in Carnot groups.

Definition 2.1 Let $1 \leq p<\infty$. We define the horizontal Sobolev space $W^{1, p}(\mathbb{G})$ as

$$
W^{1, p}(\mathbb{G})=\left\{f \in L^{p}(\mathbb{G}) \mid X_{i} f \in L^{p}(\mathbb{G}), i=1, \ldots, m\right\} .
$$

endowed with the norm

$$
\|f\|_{W^{1, p}}=\|f\|_{L^{p}(\mathbb{G})}+\left\|\nabla_{H} f\right\|_{L^{p}(\mathbb{G})} .
$$

We also define $\dot{W}^{1, p}(\mathbb{G})$ as the closure of $C_{0}^{\infty}(\mathbb{G})$ in the norm

$$
\|f\|_{1, p}=\left\|\nabla_{H} f\right\|_{L^{p}(\mathbb{G})} .
$$

Finally, $B V(\mathbb{G})$ denotes the set of functions $f \in L^{1}(\mathbb{G})$ such that

$$
\left|D_{\mathbb{G}} f\right|(\mathbb{G}):=\sup \left\{\int_{\mathbb{G}} f \operatorname{div}_{\mathbb{G}} \varphi d x \mid \varphi \in C_{c}^{1}\left(\mathbb{G}, \mathbb{R}^{m}\right),\|\varphi\|_{\infty} \leq 1\right\}
$$

is finite. Moreover, if the characteristic function $\chi_{E}$ of the measurable set $E \subset \mathbb{G}$ belongs to $B V(\mathbb{G})$ we say that $E$ has finite intrinsic perimeter and we write $\operatorname{Per}_{\mathbb{G}}(E)$ instead of $\left|D_{\mathbb{G}} \chi_{E}\right|(\mathbb{G})$,

The following results are well-known, we refer to [21, 24] for a proof.

Theorem 2.1 For any $f \in B V(\mathbb{G})$, the following identity holds:

$$
\left|D_{\mathbb{G}} f\right|(\mathbb{G})=\int_{\mathbb{R}} \operatorname{Per}_{\mathbb{G}}(\{x \in \Omega \mid f(x)>t\}) d t
$$


Theorem 2.2 Let $f \in B V(\mathbb{G})$. Then there exists a sequence $\left(f_{k}\right)_{k \in \mathbb{N}} \subset C^{\infty}(\mathbb{G})$ such that

1. $f_{k} \rightarrow f$ in $L^{1}(\mathbb{G})$;

2. $\left|D_{\mathbb{G}} f_{k}\right|(\mathbb{G}) \rightarrow\left|D_{\mathbb{G}} f\right|(\mathbb{G})$.

We conclude this section recalling the definition of horizontal fractional Sobolev spaces

Definition 2.2 Let $0<s<1$ and $1 \leq p<\infty$. We define $\dot{W}^{s, p}(\mathbb{G})$ as the closure of $C_{c}^{\infty}(\mathbb{G})$ under the norm

$$
\|f\|_{\dot{W}^{s, p}}=\left(\int_{\mathbb{G}} \int_{\mathbb{G}} \frac{|f(x)-f(y)|^{p}}{\left|y^{-1} x\right|^{Q+s p}} d x d x y\right)^{\frac{1}{p}}
$$

\section{$3 \quad$ Real Interpolation Theory and the K-method}

In this section we recall some important notions in interpolation theory, we refer the interested reader to [4] for a nice introduction to the subject.

We will use the following notations: given two quantities $f$ and $g$,

- We say that $f \lesssim g$ if there exists $C>0$ such that $f \leq C g$.

- We say that $f \approx g$ if $f \lesssim g$ and $g \lesssim f$.

For the rest of the paper, the constant $C$ with only depend of the data of $\mathbb{G}$ and on $1 \leq p<\infty$.

Let $\left(A,\|\cdot\|_{A}\right)$ and $\left(B,\|\cdot\|_{B}\right)$ be Banach spaces both continuously embedded in some Banach space $C$. We refer to the couple $(A, B)$ as an interpolation pair. Let us consider the Banach spaces $\left(A \cap B,\|\cdot\|_{A \cap B}\right)$ and $\left(A+B,\|\cdot\|_{A+B}\right)$ where

$$
\|a\|_{A \cap B}:=\max \left\{\|a\|_{A},\|a\|_{B}\right\}
$$

and

$$
\|a\|_{A+B}:=\inf _{a=a_{1}+a_{2}}\left(\left\|a_{1}\right\|_{A}+\left\|a_{2}\right\|_{B}\right) .
$$

The space $A+B$ can be equivalently renormed by Peetre's K-functional,

$$
K(t, a)=K(t, a, A, B):=\inf _{a=a_{1}+a_{2}}\left(\left\|a_{1}\right\|_{A}+t\left\|a_{2}\right\|_{B}\right) .
$$

for any $t>0$. A vector space $D$ is called intermediate if $A \cap B \subset D \subset A+B$ and the inclusions are continuous embeddings if $D$ is topologized. An intermediate space $D$ is an interpolation space is all linear operator on $A \cap B$ which map $A$ continuously into itself and $B$ continuously into itself also map $D$ into itself.

Let $(A, B)$ be an interpolation pair and let $0<s<1$ and $q \in[1, \infty)$. We define the interpolation space

$$
(A, B)_{s, q}:=\left\{f \in A+\left.B|| f\right|_{s, q}<\infty\right\}
$$

where

$$
|f|_{s, q}:=s^{\frac{1}{q}}(1-s)^{\frac{1}{q}} q^{\frac{1}{q}}\left(\int_{0}^{\infty}\left(t^{-s} K(t, f, A, B)\right)^{q} \frac{d t}{t}\right)^{\frac{1}{q}} .
$$

Definition 3.1 We say that an interpolation pair $(A, B)$ is normal if the following condition holds: 
1. $\lim _{t \rightarrow 0} \frac{K(t, f, A, B)}{t}=\|f\|_{B}$, for $f \in B$;

2. $\lim _{t \rightarrow \infty} K(t, f, A, B)=\|f\|_{A}$, for $f \in A$;

The equalities above can be relaxed to $\approx$.

The following Theorem has been proved in [29, Theorem 1]

Theorem 3.1 Let $(A, B)$ be an interpolation pair. Then

1. For $1 \leq q<\infty$ and $f \in A \cap B$ we have $\lim _{s \rightarrow 1}[f]_{s, q}=\|f\|_{B}$;

2. For $1 \leq q<\infty$ and $f \in A \cap B$ we have $\lim _{s \rightarrow 0}[f]_{s, q}=\|f\|_{A}$;

3. For $1 \leq q<\infty$ and $f \in A \cap \bigcup_{s \in(0,1)}(A, B)_{s, q}$ we have $\lim _{s \rightarrow 0}[f]_{s, q}=\|f\|_{A}$.

\section{The Modulus of Continuity}

Consider a Carnot group $\mathbb{G}$ of homogeneous dimension $Q$ and let $V=V_{1}$ be its first layer. Given $f \in L^{p}(\mathbb{G})$, where $1 \leq p<\infty$ is fixed, we define the modulus of continuity $\omega_{p}(f, t)$ by

$$
\omega_{p}(f, t)=\sup _{X \in V ;|X| \leq t}\left(\int_{\mathbb{G}}\left|f\left(e^{X} x\right)-f(x)\right|^{p} d x\right)^{\frac{1}{p}}=\sup _{y \in \exp (V) ;|y| \leq t}\left(\int_{\mathbb{G}}|f(y x)-f(x)|^{p} d x\right)^{\frac{1}{p}} .
$$

We set $\Delta_{X} f(x)=f\left(e^{X} x\right)-f(x)$ and if $h=e^{X}$ then we write $\Delta_{h} f(x)=f(h x)-f(x)$.

Proposition 4.1 Given $1 \leq p<\infty$, we have

$$
K\left(t, f, L^{p}, W^{1, p}\right) \approx \omega_{p}(f, t)+\min (1, t)\|f\|_{L^{p}} .
$$

Proof: For the sake of notation we will write $K(t, f)$ instead of $K\left(t, f, L^{p}, W^{1, p}\right)$. Recall that

$$
K(t, f)=\inf \left\{\left\|f_{0}\right\|_{L^{p}}+t\left\|f_{1}\right\|_{W^{1, p}} ; f_{0} \in L^{p}, f_{1} \in W^{1, p} ; f=f_{0}+f_{1}\right\} .
$$

It follows then that

$$
\min (1, t)\|f\|_{L^{p}} \leq\left\|f_{0}\right\|_{L^{p}}+t\left\|f_{1}\right\|_{L^{p}} \leq\left\|f_{0}\right\|_{L^{p}}+t\left\|f_{1}\right\|_{W^{1, p}}
$$

Thus,

$$
\min (1, t)\|f\|_{L^{p}} \leq K(t, f) .
$$

Let $X \in V$ with $|X| \leq t$. One can easily see that

$$
\left\|\Delta_{X} f\right\|_{L^{p}} \leq\left\|\Delta_{X} f_{0}\right\|_{L^{p}}+\left\|\Delta_{X} f_{1}\right\|_{L^{p}} \leq 2\left\|f_{0}\right\|_{L^{p}}+\left\|\Delta_{X} f_{1}\right\|_{L^{p}} .
$$

Also we have that

$$
\begin{aligned}
\Delta_{X} f_{1}(x) & =\int_{0}^{1} \frac{\partial}{\partial r} f_{1}\left(e^{r X} x\right) d r \\
& =\int_{0}^{1}\left(X f_{1}\right)\left(e^{r X} x\right) d r
\end{aligned}
$$

Hence,

$$
\left\|\Delta_{X} f_{1}(x)\right\|_{L^{p}} \leq|X|\left\|\nabla_{H} f_{1}\right\|_{L^{p}} \leq t\left\|\nabla_{H} f_{1}\right\|_{L^{p}} .
$$


Therefore, by (4.1) and (4.2)

$$
\omega_{p}(f, t)+\min (1, t)\|f\|_{L^{p}} \lesssim K(t, f) .
$$

We move now to the proof of the reverse inequality:

We identify $V$ to $\mathbb{R}^{k}$ via the Jacobian basis $\left(X_{1}, \ldots, X_{k}\right)$ and let $U$ be its unit square. That is every vector $X$ in $V$ will be written as $X=\sum_{i=1}^{k} a_{i} X_{i}$ for some $a_{i} \in \mathbb{R}$. if $X$ is in the unit square then $0 \leq a_{i} \leq 1$. Let $t>0$ and consider the function

$$
f_{0}(x)=-\int_{[0,1]^{k}} \Delta_{t \sum_{i=1}^{k} a_{i} X_{i}} f(x) d a_{1} \cdots d a_{k} .
$$

Then one have

$$
\left\|f_{0}\right\|_{L^{p}} \leq \omega_{p}(f, t) .
$$

On the other hand, we have $f_{1}=f-f_{0}=\int_{[0,1]^{k}} f\left(e^{t \sum_{i=1}^{k} a_{i} X_{i}} x\right) d a_{1} \cdots d a_{k}$. Therefore,

$$
\left\|f_{1}\right\|_{L^{p}} \leq\|f\|_{L^{p}}
$$

Next, we write $\hat{X}_{j}=\sum_{i=1, h \neq j}^{k} a_{i} X_{i}$. Notice that

$$
\frac{\partial}{\partial a_{j}}\left(f\left(e^{\sum_{i=1}^{k} t a_{i} X_{i}} x\right)\right)=t\left(X_{j} f\right)\left(e^{\sum_{i=1}^{k} t a_{i} X_{i}} x\right) .
$$

Hence,

$$
\begin{aligned}
X_{j} f_{1} & =\int_{[0,1]^{k}}\left(X_{j} f\right)\left(e^{t X} x\right) d a_{1} \cdots d a_{k} \\
& =\int_{[0,1]^{k-1}} \int_{0}^{1} \frac{1}{t} \frac{\partial}{\partial a_{j}}\left(f\left(e^{t X} x\right)\right) d a_{j} d a_{1} \cdots d a_{j-1} d a_{j+1} \cdots d a_{k} \\
& =\frac{1}{t} \int_{[0,1]^{k-1}} f\left(e^{t X} x\right)-f\left(e^{t \hat{X}_{j}} x\right) d a_{1} \cdots d a_{j-1} d a_{j+1} \cdots d a_{k} \\
& =\frac{1}{t} \int_{[0,1]^{k-1}} \Delta_{t X_{j}} f\left(e^{t \hat{X}_{j}} x\right) d a_{1} \cdots d a_{j-1} d a_{j+1} \cdots d a_{k} .
\end{aligned}
$$

Hence,

$$
\left\|\nabla_{H} f_{1}\right\|_{L^{p}} \lesssim \frac{1}{t} \omega_{p}(f, t)
$$

leading to

$$
K(t, f) \lesssim \min (1, t)\|f\|_{L^{p}}+\omega_{p}(f, t)
$$

It is easy to see from the proof above that

$$
\dot{K}(t, f)=K\left(t, f, L^{p}, \dot{W}^{1, p}\right) \approx \omega_{p}(f, t) .
$$

Here the interpolation is to be understood for the pair $\left(L^{p}, \dot{W}^{1, p}\right)$ modulo constants, which makes them two Banach spaces. 
Lemma 4.1 Define the total modulus $\bar{\omega}_{p}$ by

$$
\bar{\omega}_{p}(f, t)=\sup _{y \in \mathbb{G} ;|y| \leq t}\left(\int_{\mathbb{G}}|f(y x)-f(x)|^{p} d x\right)^{\frac{1}{p}} .
$$

Then there exists $A>0$ and $C>0$ such that

$$
\omega_{p}(f, t) \leq \bar{\omega}_{p}(f, t) \leq C \omega_{p}(f, A t) .
$$

In particular

$$
K(f, t) \approx \bar{\omega}_{p}(f, t)+\min (1, t)\|f\|_{L^{p}}
$$

Proof: First notice that

$$
\omega_{p}(f, t) \leq \bar{\omega}_{p}(f, t) .
$$

Next, we recall that, there exists $A>0$ and $N \in \mathbb{N}$ such that for all $y \in \mathbb{G}$, there exists $y_{1}, \cdots, y_{N} \in \exp (V)$ such that $y=y_{1} y_{2} \cdots y_{N}$ and $\left|y_{j}\right| \leq A|y|$ for $1 \leq j \leq N$. Thus we write

$$
|f(y x)-f(x)| \leq\left|f\left(y_{1} \cdots y_{N} x\right)-f\left(y_{2} \cdots y_{N} x\right)\right|+\cdots+\left|f\left(y_{N} x\right)-f(x)\right| .
$$

Hence,

$$
\|f(y x)-f(x)\|_{L^{p}} \leq \sum_{j=1}^{N}\left\|f\left(y_{j} x\right)-f\left(y_{j}\right)\right\|_{L^{p}} .
$$

Passing to the sup in the previous inequality gives

$$
\bar{\omega}_{p}(f, t) \leq N \omega_{p}(f, A t) .
$$

Proposition 4.2 Given $f \in L^{p}(\mathbb{G})$. then

$$
\bar{\omega}_{p}(f, t) \approx\left(\frac{1}{t^{Q}} \int_{|h| \leq t}\left\|\Delta_{h} f\right\|_{L^{p}}^{p} d h\right)^{\frac{1}{p}} .
$$

Proof: Let $\eta \in C_{c}^{\infty}\left(B_{1}\right)$ such that $\int_{\mathbb{G}} \eta=1$. We write

$$
\begin{aligned}
f(x) & =\frac{1}{t^{Q}}\left(\int_{B_{t}} f(y x) \eta\left(\delta_{t^{-1}} y\right) d y+\int_{B_{t}}(f(x)-f(y x)) \eta\left(\delta_{t^{-1}} y\right) d y\right) \\
& =I_{1}(x, t)+I_{2}(x, t) .
\end{aligned}
$$

It follows that

$$
|f(h x)-f(x)| \leq\left|I_{1}(h x, t)-I_{1}(x, t)\right|+\left|I_{2}(h x, t)\right|+\left|I_{2}(x, t)\right| .
$$

Now,

$$
\left|I_{2}(h x, t)\right|+\left|I_{2}(x, t)\right| \lesssim \frac{\|\eta\|_{\infty}}{t^{Q}} \int_{B_{t}}\left|\Delta_{y} f(x)\right|+\left|\Delta_{y} f(h x)\right| d y .
$$

On the other hand, we notice that

$$
\begin{aligned}
\nabla_{H} I_{1}(x, t) & =-\frac{1}{t^{Q+1}} \int_{B_{t}(x)} f(y) \nabla_{H} \eta\left(y x^{-1}\right) d y \\
& =-\frac{1}{t^{Q+1}} \int_{B_{t}(x)}(f(y)-f(x)) \nabla_{H} \eta\left(y x^{-1}\right) d y .
\end{aligned}
$$


But, if $h=e^{X}$ and $X \in V$, we have

$$
\begin{aligned}
I_{1}(h x, t)-I_{1}(x) & \lesssim \int_{0}^{1}|h|\left|\nabla_{H} I_{1}\right|\left(e^{r X} x, t\right) d r \\
& \lesssim|h| \frac{\left\|\nabla_{H} \eta\right\|_{\infty}}{t^{Q+1}} \int_{0}^{1} \int_{B_{t}\left(e^{r X} x\right)}\left|f(y)-f\left(e^{r X} x\right)\right| d y d r \\
& \lesssim|h| \frac{\left\|\nabla_{H} \eta\right\|_{\infty}}{t^{Q+1}} \int_{0}^{1} \int_{B_{t}}\left|f\left(y e^{r X} x\right)-f\left(e^{r X} x\right)\right| d y d r .
\end{aligned}
$$

Taking $|h| \leq t$, we get

$$
|u(h x)-u(x)| \lesssim \frac{1}{t^{Q}}\left(\int_{B_{t}}\left|\Delta_{y} f(x)\right|+\left|\Delta_{y} f(h x)\right| d y+\int_{0}^{1} \int_{B_{t}}\left|\Delta_{y} f\left(e^{r X} x\right)\right| d y d r\right) .
$$

Using Minkowski's inequality and Hölder inequality, we have

$$
\begin{aligned}
\left(\int_{G}|u(h x)-u(x)|^{p} d x\right)^{\frac{1}{p}} & \lesssim \frac{1}{t^{Q}} \int_{B_{t}}\left\|\Delta_{y} f\right\|_{L^{p}} d y \\
& \lesssim \frac{t^{\frac{Q}{p^{p}}}}{t^{Q}}\left(\int_{B_{t}}\left\|\Delta_{y} f\right\|_{L^{p}}^{p} d y\right)^{\frac{1}{p}} \\
& \lesssim\left(\frac{1}{t^{Q}} \int_{B_{t}}\left\|\Delta_{y} f\right\|_{L^{p}}^{p} d y\right)^{\frac{1}{p}}
\end{aligned}
$$

This yields to

$$
\omega_{p}(f, t) \lesssim\left(\frac{1}{t^{Q}} \int_{|h| \leq t}\left\|\Delta_{h} f\right\|_{L^{p}}^{p} d h\right)^{\frac{1}{p}}
$$

Thus,

$$
\bar{\omega}_{p}(f, t) \lesssim\left(\frac{1}{t^{Q}} \int_{|h| \leq t}\left\|\Delta_{h} f\right\|_{L^{p}}^{p} d h\right)^{\frac{1}{p}}
$$

The reverse inequality is straight forward.

Corollary 4.1 Given $0<s<1$ and $1 \leq p<\infty$, then

$$
\left(L^{p}(\mathbb{G}), \dot{W}^{1, p}(\mathbb{G})\right)_{s, p}=\dot{W}^{s, p}(\mathbb{G}) .
$$

Proof: Recall that the norm in $\left(L^{p}(\mathbb{G}), \dot{W}^{1, p}(\mathbb{G})\right)_{s, p}$ is defined by

$$
\|f\|_{s, p}=\left(\int_{0}^{\infty}\left(t^{-s} \dot{K}(t, f)\right)^{p} \frac{d t}{t}\right)^{\frac{1}{p}} .
$$

Therefore, from the previous propositions, we have that

$$
\begin{aligned}
\|f\|_{s, p} & \approx\left(\int_{0}^{\infty} \frac{1}{t^{Q+s p}} \int_{|h| \leq t} \int_{\mathbb{G}}|f(h x)-f(x)|^{p} d x d h \frac{d t}{t}\right)^{\frac{1}{p}} \\
& \approx\left(\int_{\mathbb{G}} \int_{\mathbb{G}} \int_{t \geq|h|} \frac{1}{t^{Q+s p+1}}|f(h x)-f(x)|^{p} d t d h d x\right)^{\frac{1}{p}} \\
& \approx\left(\frac{1}{Q+s p} \int_{\mathbb{G}} \int_{\mathbb{G}} \frac{|f(h x)-f(x)|^{p}}{|h|^{Q+s p}} d h d x\right)^{\frac{1}{p}}=\frac{1}{(Q+s p)^{\frac{1}{p}}}\|f\|_{\dot{W}^{s, p}} .
\end{aligned}
$$

The following Corollary is well known in the Euclidean case [14, p. 216-217] or [29, Remark 2]. We add it here in our setting. 
Corollary 4.2 Given $0<s<1$, then

$$
\left(L^{1}(\mathbb{G}), \dot{B V}(\mathbb{G})\right)_{s, 1}=\dot{W}^{s, 1}(\mathbb{G}) .
$$

Proof: Let $A_{0}=\dot{W}^{1,1}(\mathbb{G})$ and $A_{1}=L^{1}(\mathbb{G})$. Following [14], we define

$$
A_{0}+\infty \cdot A_{1}=\left\{a \in A_{0}+A_{1} \mid\|a\|_{A_{0}+\infty \cdot A_{1}}=\lim _{t \rightarrow \infty} K\left(t, a ; A_{0}, A_{1}\right)<\infty\right\}
$$

and

$$
A_{1}+\infty \cdot A_{0}=\left\{a \in A_{0}+A_{1} \mid\|a\|_{A_{1}+\infty \cdot A_{0}}=\lim _{t \rightarrow 0} \frac{1}{t} K\left(t, a ; A_{0}, A_{1}\right)<\infty\right\} .
$$

By [14, Lemma 1] and Theorem 2.2 we immediately get

$$
A_{0}+\infty \cdot A_{1}=B V(\mathbb{G}) \cap L^{1}(\mathbb{G}) \quad \text { and } \quad A_{1}+\infty \cdot A_{0}=L^{1}(\mathbb{G}),
$$

and by [14, Lemma 2] we have

$$
K\left(t, f, L^{1}, \dot{W}^{1,1}\right)=K\left(t, f, L^{1}, \dot{B V}\right)
$$

and the thesis follows by Corollary 4.1 .

Lemma 4.2 The pair $\left(L^{p}(\mathbb{G}), \dot{W}^{1, p}(\mathbb{G})\right)$ is normal.

Proof: First we show that

$$
\lim _{t \rightarrow \infty} \dot{K}(f, t) \approx\|f\|_{L^{p}}
$$

Indeed, consider a function $f_{\varepsilon}$ compactly supported such that $\left\|f-f_{\varepsilon}\right\|_{L^{p}}<\varepsilon$. Clearly for $t$ large enough, we have that

$$
\bar{\omega}_{p}\left(f_{\varepsilon}, t\right)=2\left\|f_{\varepsilon}\right\|_{L^{p}} \geq 2\|f\|_{L^{p}}-2 \varepsilon .
$$

Also, for all $f \in L^{p}$,

$$
\bar{\omega}_{p}(f, t) \leq 2\|f\|_{L^{p}}
$$

Now notice that for $h$ outside the support of $f_{\varepsilon}$

$$
\begin{aligned}
\left\|\Delta_{h} f\right\|_{L^{p}} & \geq\left\|\Delta_{h} f_{\varepsilon}\right\|_{L^{p}}-\left\|\Delta_{h}\left(f-f_{\varepsilon}\right)\right\|_{L^{p}} \\
& \geq 2\|f\|_{L^{p}}-4 \varepsilon
\end{aligned}
$$

which yields to our conclusion.

Next we need to show that

$$
\lim _{t \rightarrow 0} \frac{\dot{K}(f, t)}{t} \approx\left\|\nabla_{H} f\right\|_{L^{p}}
$$

First, we have, if $h=e^{t X}, X \in V$ then

$$
\Delta_{h} f(x)=\int_{0}^{1}(X f)\left(e^{r X} x\right) d r
$$

It follows that

$$
\omega_{p}(f, t) \leq t\left\|\nabla_{H} f\right\|_{L^{p}} .
$$

Now, let $f_{\varepsilon} \in C_{c}^{\infty}(\mathbb{G})$ such that $\left\|\nabla_{H}\left(f-f_{\varepsilon}\right)\right\|_{L^{p}}<\varepsilon$. Notice that

$$
f_{\varepsilon}(h x)-f_{\varepsilon}(x)-X f_{\varepsilon}(x)=\int_{0}^{1}\left(X f_{\varepsilon}\right)\left(e^{r X} x\right)-\left(X f_{\varepsilon}\right)(x) d r .
$$


Hence, one can see that

$$
\mu_{\varepsilon}(t)=\sup _{|X|=t, h=e^{X}} \frac{\left\|f_{\varepsilon}(h x)-f_{\varepsilon}(x)-\left(X f_{\varepsilon}\right)(x)\right\|_{L^{p}}}{t} \rightarrow 0, \quad t \rightarrow 0 .
$$

In particular, there exists $t_{\varepsilon}$ small, so that $\mu_{\varepsilon}(t)<\varepsilon$ for $0<t<t_{\varepsilon}$. Moreover, we have that

$$
\omega_{p}\left(f_{\varepsilon}, t\right) \leq \omega_{p}\left(f_{\varepsilon}\right)+t \varepsilon
$$

Hence, for $0<t<t_{\varepsilon}$ we have that

$$
\left\|\nabla_{H} f\right\|_{L^{p}} \leq\left\|\nabla_{H} f_{\varepsilon}\right\|_{L^{p}}+\varepsilon \leq \mu_{\varepsilon}(t)+\frac{\omega_{p}\left(f_{\varepsilon}, t\right)}{t}+\varepsilon \leq \frac{\omega_{p}(f, t)}{t}+2 \varepsilon .
$$

and this finishes the proof.

The following result immediately follows from Lemma 4.2 and the fact that $K\left(t, f, L^{1}, \dot{W}^{1,1}\right)=$ $K\left(t, f, L^{1}, \dot{B} V\right)$.

Corollary 4.3 The pair $\left(L^{1}(\mathbb{G}), \dot{B V}(\mathbb{G})\right)$ is normal.

\section{Applications}

Applying Lemma 4.2 and Corollary 4.3 we get various interesting limiting formulas. Theorems 5.1 and 5.2 can be proved exactly as in [29] whereas Theorem 5.3 has its roots in $[5,15,33]$.

\subsection{Limit Behavior of Fractional Sobolev Spaces}

Theorem 5.1 (Bourgain-Brézis-Mironescu) Let $f \in \dot{W}^{1, p}(\mathbb{G})$, then

$$
\lim _{s \rightarrow 1}(1-s)^{\frac{1}{p}}\left(\int_{\mathbb{G}} \int_{\mathbb{G}} \frac{|f(x)-f(y)|^{p}}{\left|y^{-1} x\right|^{Q+s p}} d x d y\right)^{\frac{1}{p}} \approx(Q+p)^{\frac{1}{p}} p^{-\frac{1}{p}}\left\|\nabla_{H} f\right\|_{L^{p}}
$$

Theorem 5.2 (Maz'ya-Shaposhnikova) Let $f \in \cup_{s \in(0,1)} \dot{W}^{s, p}$, then

$$
\lim _{s \rightarrow 0} s^{\frac{1}{p}}\left(\int_{\mathbb{G}} \int_{\mathbb{G}} \frac{|f(x)-f(y)|^{p}}{\left|y^{-1} x\right|^{Q+s p}} d x d y\right)^{\frac{1}{p}} \approx Q^{\frac{1}{p}} p^{-\frac{1}{p}}\|f\|_{L^{p}}
$$

Theorem 5.3 (Davila) Let $f \in \dot{B V}(\mathbb{G})$, then

$$
\lim _{s \rightarrow 1}(1-s) \int_{\mathbb{G}} \int_{\mathbb{G}} \frac{|f(x)-f(y)|}{\left|y^{-1} x\right|^{Q+s}} d x d y \approx(Q+1)\left|D_{\mathbb{G}} f\right|(\mathbb{G}) .
$$

\subsection{Fractional Perimeter and its Limiting Behavior}

We recall here the definition of the fractional perimeter $\operatorname{Per}_{s}$ in a Carnot group $\mathbb{G}$, namely

$$
\operatorname{Per}_{s}(A)=\int_{A} \int_{\mathbb{G} \backslash A} \frac{1}{\left|y^{-1} x\right|^{Q+s}} d y d x \quad \text { for } A \subset \mathbb{G} .
$$

A similar definition has been proposed in [16], moreover if $\mathbb{G}=\mathbb{R}^{n}$ the previous definition boils to the one proposed by Caffarelli-Roquejoffre and Savin in [12]. 
Clearly, $\operatorname{Per}_{s}(A)=\left\|\chi_{A}\right\|_{\dot{W}^{1, s}}$ and from the results above, we have that

$$
\lim _{s \rightarrow 1}(1-s) \operatorname{Per}_{s}(A) \approx \operatorname{Per}_{\mathbb{G}}(A)
$$

and

$$
\lim _{s \rightarrow 0} s \operatorname{Per}_{s}(A) \approx|A| .
$$

In order to provide a more exact convergence, as in [33], we define the following perimeter:

$$
\widetilde{\operatorname{Per}}_{s}(A)=s(1-s) \int_{0}^{\infty} t^{-s} K\left(t, \chi_{A}, L^{1}, \dot{B V}\right) \frac{d t}{t}
$$

Then we have

Theorem 5.4 Let $A \subset \mathbb{G}$, then the we have

$$
\widetilde{\operatorname{Per}}_{s}(A) \leq|A|^{1-s} \operatorname{Per}_{\mathbb{G}}(A)^{s}
$$

Moreover,

i) $\lim _{s \rightarrow 0} \widetilde{\operatorname{Per}}_{s}(A)=|A|$.

ii) $\lim _{s \rightarrow 1} \widetilde{\operatorname{Per}}_{s}(A)=\operatorname{Per}_{\mathbb{G}}(A)$.

Proof: For short we will write $K\left(t, \chi_{A}, L^{1}, B V\right)=K(t, A)$. Notice that $i$ ) and $\left.i i\right)$ follow from the interpolation limits as above. So we propose to establish the first statement. By the definition of $K$, we have that

$$
K(t, A) \leq|A| \quad \text { and } \quad K(t, A) \leq t \operatorname{Per}_{\mathbb{G}}(A)
$$

Therefore,

$$
\begin{aligned}
\int_{0}^{\infty} t^{-s} K(t, A) \frac{d t}{t} & =\int_{0}^{r} t^{-s} K(t, A) \frac{d t}{t}+\int_{r}^{\infty} t^{-s} K(t, A) \frac{d t}{t} \\
& \leq \operatorname{Per}_{\mathbb{G}}(A) \int_{0}^{r} t^{-s} d s+|A| \int_{r}^{\infty} t^{-s-1} d t \\
& \leq \operatorname{Per}_{\mathbb{G}}(A) \frac{r^{1-s}}{1-s}+|A| r^{-s} s .
\end{aligned}
$$

Minimizing over $r \in(0, \infty)$ we get the desired inequality.

Following [33], we provide another quantitative form of $K$ using the symmetric difference of sets.

Proposition 5.1 Let $A \subset \mathbb{G}$, then

$$
K(t, A)=\inf _{U \subset \mathbb{G}}|A \Delta U|+t \operatorname{Per}_{\mathbb{G}}(U) .
$$

Proof: Let $g(t, A)=\inf _{U \subset \mathbb{G}}|A \Delta U|+t P e r_{\mathbb{G}}(U)$. First, we write $\chi_{A}=\chi_{A}-\chi_{U}+\chi(U)$. Since $\left\|\chi_{A}-\chi_{U}\right\|_{L^{1}}=|A \Delta U|$ and $\left|D_{G} \chi_{U}\right|(\mathbb{G})=\operatorname{Per}_{\mathbb{G}}(U)$, it follows from the definition of $K$ that

$$
K(t, A) \leq g(t, A)
$$


On the other hand, we claim first that

$$
K(t, f)=\inf \left\{\left\|f_{1}\right\|_{L^{1}}+t\left\|\nabla_{H} f_{2}\right\|_{L^{1}} ; f=f_{1}+f_{2}, f_{1} \in L^{1}(\mathbb{G}) ; f_{2} \in C_{c}^{\infty}(\mathbb{G})\right\} .
$$

Indeed, we already have from the definition itself that

$$
K(t, f) \leq \inf \left\{\left\|f_{1}\right\|_{L^{1}}+t\left\|\nabla_{H} f_{2}\right\|_{L^{1}} ; f=f_{1}+f_{2}, f_{1} \in L^{1}(\mathbb{G}) ; f_{2} \in C_{c}^{\infty}(\mathbb{G})\right\} .
$$

Now given $f=f_{1}+f_{2}$ such that $f_{1} \in L^{1}$ and $f_{2} \in \dot{B V}$ with

$$
\left\|f_{1}\right\|_{L^{1}}+t\left|D_{\mathbb{G}} f_{2}\right|(\mathbb{G}) \leq K(t, f)+\varepsilon,
$$

then by Theorem 1.2, we can always find $g_{\varepsilon} \in C_{c}^{\infty}(\mathbb{G})$ such that $\left|D_{\mathbb{G}}\left(f_{2}-g_{\varepsilon}\right)\right|(\mathbb{G})<\frac{\varepsilon}{t}$ and $\left\|f_{2}-g_{\varepsilon}\right\|_{L^{1}}<\varepsilon$. Hence, writing $f=f_{1}+f_{2}-g_{\varepsilon}+g_{\varepsilon}$ we get

$$
\left\|f_{1}+f_{2}-g_{\varepsilon}\right\|_{L^{1}}+t\left|D_{\mathbb{G}} g_{\varepsilon}\right|(\mathbb{G}) \leq\left\|f_{1}\right\|_{L^{1}}+t\left|D_{\mathbb{G}} f_{2}\right|(\mathbb{G})+2 \varepsilon \leq K(t, f)+3 \varepsilon .
$$

Therefore,

$$
\inf \left\{\left\|f_{1}\right\|_{L^{1}}+t\left\|\nabla_{H} f_{2}\right\|_{L^{1}} ; f=f_{1}+f_{2}, f_{1} \in L^{1}(\mathbb{G}) ; f_{2} \in C_{c}^{\infty}(\mathbb{G})\right\} \leq K(t, f) .
$$

Now, we can decompose the characteristic function of $A$ as follows: $\chi_{A}=\chi_{A}-f+f$ where $f \in C_{c}^{\infty}(\mathbb{G})$. Let $U=\{|f|>r\}$, then we have

$$
A \Delta U \subset\left(\left\{\left|\chi_{A}-f\right| \geq r\right\} \backslash A\right) \cup\left(\left\{\left|\chi_{A}-f\right| \geq 1-r\right\} \cap A\right) .
$$

Using Theorem 1.1, we have

$$
\int_{0}^{\infty}\left|\left\{\left|\chi_{A}-f\right| \geq r\right\}\right| d r+t \int_{0}^{\infty} \operatorname{Per}_{\mathbb{G}}(\{|f|>r\}) d r=\left\|\chi_{A}-f\right\|_{L^{1}}+t\left|D_{\mathbb{G}} f\right|(\mathbb{G}) .
$$

Next, we notice that

$$
\int_{0}^{1}\left(\left|\left\{\left|\chi_{A}-f\right| \geq r\right\} \backslash A\right|+\left|\left\{\left|\chi_{A}-f\right| \geq 1-r\right\} \cap A\right|\right) d r=\int_{0}^{1}\left|\left\{\left|\chi_{A}-f\right| \geq r\right\}\right| d r \leq \int_{0}^{\infty}\left|\left\{\left|\chi_{A}-f\right| \geq r\right\}\right| d r .
$$

Hence,

$$
\int_{0}^{1}\left(\left|\left\{\left|\chi_{A}-f\right| \geq r\right\} \backslash A\right|+\left|\left\{\left|\chi_{A}-f\right| \geq 1-r\right\} \cap A\right|+\operatorname{Per}_{\mathbb{G}}(\{|f| \geq r\})\right) d r \leq\left\|\chi_{A}-f\right\|_{L^{1}}+t\left|D_{\mathbb{G}} f\right|(\mathbb{G}) .
$$

Therefore, by the mean value theorem, there exists $r \in[0,1]$, depending on $t$ such that

$$
\left(\left|\left\{\left|\chi_{A}-f\right| \geq r\right\} \backslash A\right|+\left|\left\{\left|\chi_{A}-f\right| \geq 1-r\right\} \cap A\right|+t \operatorname{Per}_{\mathbb{G}}(\{|f| \geq r\})\right) \leq\left\|\chi_{A}-f\right\|_{L^{1}}+t\left|D_{\mathbb{G}} f\right|(\mathbb{G}) .
$$

In particular,

$$
\begin{aligned}
g(t, A) & \leq|A \Delta U|+t \operatorname{Per}_{\mathbb{G}}(U) \leq\left(\left|\left\{\left|\chi_{A}-f\right| \geq r\right\} \backslash A\right|+\left|\left\{\left|\chi_{A}-f\right| \geq 1-r\right\} \cap A\right|+t \operatorname{Per}_{\mathbb{G}}(\{|f| \geq r\})\right) \\
& \leq\left\|\chi_{A}-f\right\|_{L^{1}}+t\left|D_{\mathbb{G}} f\right|(\mathbb{G}) .
\end{aligned}
$$

Leading to

$$
g(t, A) \leq K(t, A) .
$$




\section{References}

[1] Ambrosio, L., De Philippis, G., Martinazzi, L.: $\Gamma$-convergence of nonlocal perimeter functionals, Manuscripta Math. 134 (2011), 377-403.

[2] Aronszajn, N.: Boundary values of functions with finite Dirichlet integral, Techn. Report of Univ. of Kansas 14 (1955), 77-94.

[3] Barbieri, D.: Approximations of Sobolev norms in Carnot groups, Comm. Contemp. Math. 13 (2011), 765-794.

[4] Bennett, C., Sharpley, R. : Interpolation of Operators, Pure and Applied Mathematics, vol.129, Academic Press, Boston, MA, USA. 1988.

[5] Bourgain, J., Brezis, H., Mironescu, P.: Another look at Sobolev spaces, in Optimal Control and Partial Differential Equations. A Volume in Honor of Professor Alain Bensoussan's 60th Birthday (eds. J. L. Menaldi, E. Rofman and A. Sulem), IOS Press, Amsterdam, 2001, 439-455.

[6] Bourgain, J., Brezis, H., Mironescu, P.: Limiting embedding theorems for $W^{s, p}$ when $s \uparrow 1$ and applications, J. Anal. Math. 87 (2002), 77-101.

[7] Brezis, H.: How to recognize constant functions. Connections with Sobolev spaces, Volume in honor of M. Vishik, Uspekhi Mat. Nauk 57 (2002), 59-74 (in Russian). English translation in Russian Math. Surveys 57 (2002), 693-708.

[8] Brezis, H. New approximations of the total variation and filters in imaging, Rend Accad. Lincei 26 (2015), 223-240.

[9] Brezis, H., Nguyen, H. M.: Non-local functionals related to the total variation and connections with Image Processing, http://arxiv.org/abs/1608.08204. To appear in Annals of PDE.

[10] Brezis, H., Nguyen, H. M.: The BBM formula revisited, Rend. Accad. Lincei 27 (2016), 515-533.

[11] Bonfiglioli, A., Lanconelli, E., Uguzzoni, U.: Stratified Lie groups and potential theory for their sub-Laplacians. Springer, 2007.

[12] Caffarelli, L., Roquejoffre, J.-M., Savin, 0.: Nonlocal minimal surfaces. Comm. Pure Appl. Math. 63 (2010), no. 9, 1111-1144.

[13] Capogna, L., Danielli, D., Pauls, S. D., Tyson, J. T.: An introduction to the Heisenberg group and the sub-Riemannian isoperimetric problem, Birkhäuser, 2007.

[14] Cwikel, M.: Monotonicity properties of interpolation spaces. Ark. Mat. 14 (1976), no. 2, 213-236.

[15] Dávila, J.: On an open question about functions of bounded variation, Calc. Var. Partial Differ. Equ. 15 (2002) 519-527. 
[16] Ferrari, F., Miranda, M.; Pallara, D.; Pinamonti, A.; Sire, Y.: Fractional laplacians, perimeters and heat semigroups in carnot groups, Discrete Contin. Dyn. Syst. Ser. S 11 (2018), no. 3, 477-491.

[17] Folland G. B.: Subelliptic estimates and function spaces on nilpotent Lie groups. Ark. Mat. 13 (1975), 161-207.

[18] Folland G. B., Stein, E. M.: Hardy spaces on homogeneous groups. Princeton University Press, (1982).

[19] Franchi, B.: BV spaces and rectifiability for Carnot-Caratheodory metrics: an introduction, VII NAFSA Spring School, Math. Inst. Acad. Sc. Czech Rep., (2003), pp. 73-132

[20] Franchi, B., Serapioni, R., Serra Cassano, F.: Rectifiability and perimeter in the Heisenberg group, Math. Ann. 321 (3) (2001) 479-531.

[21] Franchi, B., Serapioni, R., Serra Cassano, F.: Meyers-Serrin type theorems and relaxation of variational integrals depending on vector fields. Houston J. Math. 22, (1996), no. 4, 859-890.

[22] Frank, R.L., del Mar González, M., Monticelli, D.D, Tan, J.: An extension problem for the CR fractional Laplacian, Adv. Math. 270 (2015), 97-137.

[23] Frank, R.L., Lieb, E.H.: Sharp constants in several inequalities on the Heisenberg group, Ann. of Math. (2) 176 (2012), no. 1, 349-381.

[24] Garofalo, N., Nhieu, D.-M.: Isoperimetric and Sobolev inequalities for CarnotCarathéodory spaces and the existence of minimal surfaces, Comm. Pure Appl. Math. 49 (1996), no. 10, 1081-1144.

[25] Gagliardo, E. : Proprietá di alcune classi di funzioni in piú variabili, Ricerche Mat. 7 (1958), 102-137.

[26] Gover, A., Graham, C.R.: CR invariant powers of the sub-Laplacian, J. Reine Angew. Math. 583 (2005), 1-27.

[27] Guidi, C., Maalaoui, A., Martino, V.: Palais-Smale sequences for the fractional CR Yamabe functional and multiplicity results, arXiv:1801.06399v1, Preprint.

[28] Maalaoui, A.: A Note on Commutators of the Fractional Sub-Laplacian on Carnot Groups, arXiv:1711.10298v1. To appear in Comm. Pure Appl. Anal.

[29] Milman, M.: Notes on limits of Sobolev spaces and the continuity of interpolation scales. Trans. Amer. Math. Soc. 357 (2005), no. 9, 3425-3442.

[30] Nguyen, H. M., Pinamonti, A., Squassina, M., Vecchi, E.: New characterization of magnetic Sobolev spaces. Adv. Nonlinear Anal. 7 (2018), no. 2, 227-245.

[31] Pinamonti, A., Squassina, M., Vecchi, E.: Magnetic BV functions and the Bourgain-Brezis-Mironescu formula. Adv. Calc. Var. (2017), https://doi.org/10.1515/acv2017-0019. 
[32] Pinamonti, A., Squassina, M., Vecchi, E.: The Maz'ya-Shaposhnikova limit in the magnetic setting. J. Math. Anal. Appl., 449 (2017), 1152-1159.

[33] Ponce, A. C., Spector, D.: A note on the fractional perimeter and interpolation. C. R. Math. Acad. Sci. Paris 355 (2017), no. 9, 960-965.

[34] Roncala, L., Thangavelu, V.: Hardy's inequality for fractional powers of the sublaplacian on the Heisenberg group. Advances in Mathematics, 302, (2016), 106-158.

[35] Saka, K.: Besov spaces and Sobolev spaces on a nilpotent Lie group, Tohoku Math. J. (2) 31 (1979), no. 4, 383-437. doi:10.2748/tmj/1178229728.

[36] Sánchez-Calle, A.: Fundamental solutions and geometry of the sum of squares of vector fields. Invent. Math. 78, (1984), 143-160.

[37] Slobodeckij, L. N.: Generalized Sobolev spaces and their applications to boundary value problems of partial differential equations, Leningrad. Gos. Ped. Inst. Ucep. Zap. 197 (1958), 54-112.

[38] Squassina, M., Volzone, B.: Bourgain-Brezis-Mironescu formula for magnetic operators C. R. Math. Acad. Sci. Paris 354 (2016), 825-831.

[39] Stein, E. M.: Harmonic Analysis: Real-Variable Methods, Orthogonality and Oscillatory Integrals. Princeton University Press, 1993. 